\title{
Impacts of dark energy on constraining neutrino mass after Planck 2018
}

\author{
Ming Zhang, ${ }^{1}$ Jing-Fei Zhang, ${ }^{1}$ and Xin Zhang*1,2,3,4, ${ }^{\dagger}$ \\ ${ }^{1}$ Department of Physics, College of Sciences, Northeastern University, Shenyang 110819, China \\ ${ }^{2}$ Ministry of Education's Key Laboratory of Data Analytics and Optimization for Smart Industry, \\ Northeastern University, Shenyang 110819, China \\ ${ }^{3}$ Center for High Energy Physics, Peking University, Beijing 100080, China \\ ${ }^{4}$ Center for Gravitation and Cosmology, Yangzhou University, Yangzhou 225009, China
}

\begin{abstract}
Considering the mass splittings of three active neutrinos, we investigate how the nature of dark energy affects the cosmological constraints on the total neutrino mass $\sum m_{\nu}$ using the latest cosmological observations. In this paper, some typical dark energy models, including $\Lambda$ CDM, $w$ CDM, CPL, and HDE models, are discussed. In the analysis, we also consider the effects from the neutrino mass hierarchies, i.e., the degenerate hierarchy $(\mathrm{DH})$, the normal hierarchy $(\mathrm{NH})$, and the inverted hierarchy ( $\mathrm{IH})$. We employ the current cosmological observations to do the analysis, including the Planck 2018 temperature and polarization power spectra, the baryon acoustic oscillations (BAO), the type Ia supernovae (SNe), and the Hubble constant $H_{0}$ measurement. In the $\Lambda \mathrm{CDM}+\sum m_{\nu}$ model, we obtain the upper limits of the neutrino mass $\sum m_{\nu}<0.123 \mathrm{eV}(\mathrm{DH}), \sum m_{\nu}<0.156$ $\mathrm{eV}(\mathrm{NH})$, and $\sum m_{\nu}<0.185 \mathrm{eV}(\mathrm{IH})$ at the $95 \%$ C.L., using the Planck+BAO+SNe data combination. For the $w \mathrm{CDM}+\sum m_{\nu}$ model and the $\mathrm{CPL}+\sum m_{\nu}$ model, larger upper limits of $\sum m_{\nu}$ are obtained compared to those of the $\Lambda \mathrm{CDM}+\sum m_{\nu}$ model. The most stringent constraint on the neutrino mass, $\sum m_{\nu}<0.080 \mathrm{eV}(\mathrm{DH})$, is derived in the $\mathrm{HDE}+\sum m_{\nu}$ model. In addition, we find that the inclusion of the local measurement of the Hubble constant in the data combination leads to tighter constraints on the total neutrino mass in all these dark energy models.
\end{abstract}

\section{INTRODUCTION}

Neutrino oscillation experiments $[1,2]$ indicate that the three neutrino flavor eigenstates $\left(\nu_{e}, \nu_{\mu}, \nu_{\tau}\right)$ are actually quantum superpositions of the three mass eigenstates $\left(\nu_{1}, \nu_{2}, \nu_{3}\right)$ with masses $m_{1}, m_{2}$, and $m_{3}$. However, neutrino oscillation experiments cannot measure the absolute neutrino masses, but can only give the squared mass differences between the different mass eigenstates of neutrino. The solar and reactor experiments gave the result of $\Delta m_{21}^{2} \simeq 7.54 \times 10^{-5} \mathrm{eV}^{2}$ and the atmospheric and accelerator beam experiments gave the result of $\left|\Delta m_{31}^{2}\right| \simeq 2.46 \times 10^{-3} \mathrm{eV}^{2}$ [3], which indicates that there are two possible neutrino mass hierarchies, i.e., the normal hierarchy (NH) with $m_{1}<m_{2} \ll m_{3}$ and the inverted hierarchy (IH) with $m_{3} \ll m_{1}<m_{2}$. The case of neglecting the neutrino mass splitting, namely $m_{1}=m_{2}=m_{3}$, is called the degenerate hierarchy (DH).

Nevertheless, cosmological observations could provide a useful tool to measure the absolute neutrino total mass. With the decrease of the neutrino temperature, neutrino becomes non-relativistic at $T \sim 0.15 \mathrm{eV}$ in the evolution of the universe. Then the mass effect of neutrinos begins to appear, which leads to a nonnegligible influence on the cosmic microwave background (CMB) and largescale structure (LSS) [4-10]. Therefore, we could extract much useful information about neutrino from cosmological observations.

Recently, the observational data of Planck 2018 have

\footnotetext{
${ }^{*}$ Corresponding author

${ }^{\dagger}$ Electronic address: zhangxin@mail.neu.edu.cn
}

been released by the Planck collaboration, and according to the latest data the limit of the total neutrino mass is $\sum m_{\nu}<0.24 \mathrm{eV}$ (95\% C.L., TT,TE,EE+lowE+lensing) [11]. Since the baryon acoustic oscillations (BAO) data at low redshifts can break the geometric degeneracy inherent in $\mathrm{CMB}$, the combination of the acoustic scales measured by the $\mathrm{CMB}$ and $\mathrm{BAO}$ data can determine the background geometry sufficiently. Combining $\mathrm{BAO}$ data with $\mathrm{CMB}$ data, the neutrino mass can be constrained to be $\sum m_{\nu}<0.12 \mathrm{eV}$ (95\% C.L., TT, TE,EE+lowE+lensing+BAO). Adding the Pantheon type Ia supernovae (SNe) luminosity distance measurements, the constraint only becomes slightly better, with the result still roughly $\sum m_{\nu}<0.11 \mathrm{eV}(95 \%$ C.L., $\mathrm{TT}, \mathrm{TE}, \mathrm{EE}+$ lowE+lensing $+\mathrm{BAO}+\mathrm{SNe}$ ). It is noted that these results are based on the $\Lambda \mathrm{CDM}+\sum m_{\nu}$ model.

Therefore, we wish to investigate the impacts of dark energy on constraining the total neutrino mass. In this work, we consider some typical dark energy models, including the $\Lambda \mathrm{CDM}$ model, the $w \mathrm{CDM}$ model, the $w_{0} w_{a} \mathrm{CDM}$ model (also known as the Chevallier-PolarskiLinder model or the CPL model) [12, 13], and the holographic dark energy (HDE) model [14-21]. In addition, we also consider the effects from the neutrino mass hierarchies (i.e., $\mathrm{DH}, \mathrm{NH}$, and $\mathrm{IH}$ ) in our analysis.

More recently, some related studies of constraints on the total neutrino mass have been made; see, e.g., Refs. [22-60]. The cosmological constraints on the total neutrino mass in dynamical dark energy models have been discussed in, e.g., Refs. [45, 46], which indicates that the nature of dark energy can have a significant influence on the measurement of the total neutrino mass. As the latest CMB data have been released by the Planck collaboration, the results need to be updated. In this work, 
we employ the latest cosmological observations, including the $\mathrm{CMB}, \mathrm{BAO}, \mathrm{SNe}$, and $H_{0}$ data to make a new analysis.

In this work, we will use the recent local measurement of the Hubble constant $H_{0}$, with the result of $H_{0}=74.03 \pm 1.42 \mathrm{~km} \mathrm{~s}^{-1} \mathrm{Mpc}^{-1}$ (68\% C.L.), by Riess et al. [61]. Note that this local measurement result is in more than $4 \sigma$ tension with the result of the Planck 2018 observation assuming a 6 -parameter base- $\Lambda$ CDM model. Thus, we also wish to investigate how the inclusion of the $H_{0}$ local measurement would affect the measurement of the total neutrino mass in these dark energy models.

This paper is organized as follows. In Sec. II, we describe the methodology in our analysis. In Sec. III, we show the results and make some discussions. Finally, the conclusion is given in Sec. IV.

\section{METHODOLOGY}

We take into account the neutrino mass splittings between the three active neutrinos. We employ the measurement results of neutrino oscillation experiments [3],

$$
\begin{gathered}
\Delta m_{21}^{2} \equiv m_{2}^{2}-m_{1}^{2}=7.54 \times 10^{-5} \mathrm{eV}^{2}, \\
\left|\Delta m_{31}^{2}\right| \equiv\left|m_{3}^{2}-m_{1}^{2}\right|=2.46 \times 10^{-3} \mathrm{eV}^{2} .
\end{gathered}
$$

The total neutrino mass $\sum m_{\nu}$ is the sum of three active neutrino mass. For the $\mathrm{NH}$ case, $\sum m_{\nu}$ is written as

$$
\sum m_{\nu}^{\mathrm{NH}}=m_{1}+\sqrt{m_{1}^{2}+\Delta m_{21}^{2}}+\sqrt{m_{1}^{2}+\Delta m_{31}^{2}},
$$

where $m_{1}$ is a free parameter. For the IH case, $\sum m_{\nu}$ is written as

$$
\sum m_{\nu}^{\mathrm{IH}}=\sqrt{m_{3}^{2}+\left|\Delta m_{31}^{2}\right|}+\sqrt{m_{3}^{2}+\left|\Delta m_{31}^{2}\right|+\Delta m_{21}^{2}}+m_{3},
$$

where $m_{3}$ is a free parameter. For the $\mathrm{DH}$ case, ignoring the neutrino mass splittings, $\sum m_{\nu}$ can be written as

$$
\sum m_{\nu}^{\mathrm{DH}}=m_{1}+m_{2}+m_{3}=3 m,
$$

where $m$ is a free parameter. Therefore, the lower bounds of $\sum m_{\nu}$ are $0 \mathrm{eV}, 0.06 \mathrm{eV}$ and $0.1 \mathrm{eV}$ for $\mathrm{DH}, \mathrm{NH}$ and $\mathrm{IH}$, respectively. In this way, the total neutrino mass $\sum m_{\nu}$ as an additional parameter will be considered in our analysis.

In this paper, we make a global fit analysis on the different dark energy models, i.e., the $\Lambda \mathrm{CDM}+\sum m_{\nu}$ model, the $w \mathrm{CDM}+\sum m_{\nu}$ model, the $\mathrm{CPL}+\sum m_{\nu}$ model, and the HDE $+\sum m_{\nu}$ model. We modify the publicly available Markov chain Monte-Carlo package CosmoMC [62] (that uses the Boltzmann solver CAMB [63]) to do the numerical calculations.

Here, we give a brief introduction to these dark energy models.
- The $\Lambda \mathrm{CDM}+\sum m_{\nu}$ model: The model containing a cosmological constant $\Lambda$ and cold dark matter is called the $\Lambda$ CDM model, which can fit various cosmological observations well. For the $\Lambda \mathrm{CDM}+\sum m_{\nu}$ model, the parameter space vector is:

$$
P_{1} \equiv\left(\omega_{b}, \omega_{c}, \Theta_{\mathrm{s}}, \tau, n_{\mathrm{s}}, \ln \left[10^{10} \mathrm{~A}_{\mathrm{s}}\right], \sum \mathrm{m}_{\nu}\right),
$$

where $\omega_{b} \equiv \Omega_{b} h^{2}$ and $\omega_{c} \equiv \Omega_{c} h^{2}$ represent baryon and cold dark matter densities, respectively, $\Theta_{\mathrm{s}}$ is the ratio between sound horizon $r_{s}$ and angular diameter distance $D_{\mathrm{A}}$ at the time of photon decoupling, $\tau$ is the optical depth to the reionization of the universe, $n_{\mathrm{s}}$ and $A_{\mathrm{s}}$ are the power-law spectral index and amplitude of the power spectrum of primordial curvature perturbations, respectively, and $\sum m_{\nu}$ is the total neutrino mass.

- The $w \mathbf{C D M}+\sum m_{\nu}$ model: The $w \mathrm{CDM}$ model is the simplest dynamical dark energy model, in which the equation-of-state (EoS) parameter $w(z)$ is assumed to be a constant. For the $w \mathrm{CDM}+\sum m_{\nu}$ model, the parameter space vector is:

$P_{2} \equiv\left(\omega_{b}, \omega_{c}, \Theta_{\mathrm{s}}, \tau, n_{\mathrm{s}}, \ln \left[10^{10} \mathrm{~A}_{\mathrm{s}}\right], w, \sum \mathrm{m}_{\nu}\right)$

- The CPL $+\sum m_{\nu}$ model: For probing the evolution of $w(z)$, the most widely used parametrization model is the CPL model (also called the $w_{0} w_{a} \mathrm{CDM}$ model) $[12,13]$. The form of $w(z)$ in this model is given by

$$
w(z)=w_{0}+w_{a}(1-a)=w_{0}+w_{a} \frac{z}{1+z},
$$

where $w_{0}$ and $w_{a}$ are free parameters. So, for the $\mathrm{CPL}+\sum m_{\nu}$ model, the parameter space vector is:

$$
P_{3} \equiv\left(\omega_{b}, \omega_{c}, \Theta_{\mathrm{s}}, \tau, n_{\mathrm{s}}, \ln \left[10^{10} \mathrm{~A}_{\mathrm{s}}\right], w_{0}, w_{a}, \sum \mathrm{m}_{\nu}\right) \text {. }
$$

- The HDE $+\sum m_{\nu}$ model: The HDE model is built based on the the effective quantum field theory together with the holographic principle of quantum gravity. We can put an energy bound on the vacuum energy density, $\rho_{\mathrm{de}} L^{3} \leq M_{\mathrm{Pl}}^{2} L$, where $M_{\mathrm{Pl}}$ is the reduced Planck mass, which means that the total energy in a spatial region with size $L$ should not exceed the mass of a black hole with the same size [64]. The largest length size that is compatible with this bound is the infrared cutoff size of this effective quantum field theory. An infrared scale can saturate that bound, and thus the dark energy density can be written as [14]

$$
\rho_{\mathrm{de}}=3 c^{2} M_{\mathrm{Pl}}^{2} L^{-2},
$$

where $c$ is a dimensionless phenomenological parameter (note that here $\mathrm{c}$ is not the speed of light), 
TABLE I: Fitting results of the cosmological parameters in the $\Lambda$ CDM $+\sum m_{\nu}$ model for three neutrino mass hierarchy cases, i.e., the $\mathrm{DH}$ case, the $\mathrm{NH}$ case, and the $\mathrm{IH}$ case, using the Planck $+\mathrm{BAO}+\mathrm{SNe}$ and Planck $+\mathrm{BAO}+\mathrm{SNe}+H_{0}$ data combinations.

\begin{tabular}{|c|c|c|c|c|c|c|}
\hline Data & \multicolumn{3}{|c|}{ Planck+BAO+SNe } & \multicolumn{3}{|c|}{$\mathrm{Planck}+\mathrm{BAO}+\mathrm{SNe}+H_{0}$} \\
\hline Mass ordering & $\mathrm{DH}$ & $\mathrm{NH}$ & $\mathrm{IH}$ & $\mathrm{DH}$ & $\mathrm{NH}$ & $\mathrm{IH}$ \\
\hline$H_{0}\left[\mathrm{~km} \mathrm{~s}^{-1} \mathrm{Mpc}^{-1}\right]$ & $67.75 \pm 0.49$ & $67.48 \pm 0.47$ & $67.26 \pm 0.45$ & $68.40 \pm 0.44$ & $68.11 \pm 0.43$ & $67.88 \pm 0.43$ \\
\hline$\Omega_{\mathrm{m}}$ & $0.3097 \pm 0.0063$ & $0.3126 \pm 0.0063$ & $0.3150 \pm 0.0060$ & $0.3015 \pm 0.0056$ & $0.3044 \pm 0.0056$ & $0.3069 \pm 0.0056$ \\
\hline$\sigma_{8}$ & $0.812_{-0.008}^{+0.013}$ & $0.801_{-0.008}^{+0.011}$ & $0.793_{-0.008}^{+0.010}$ & $0.813_{-0.008}^{+0.010}$ & $0.801_{-0.008}^{+0.009}$ & $0.792_{-0.008}^{+0.009}$ \\
\hline$\sum m_{\nu}[\mathrm{eV}]$ & $<0.123$ & $<0.156$ & $<0.185$ & $<0.082$ & $<0.125$ & $<0.160$ \\
\hline$\chi^{2}$ & 3805.133 & 3807.205 & 3809.012 & 3821.466 & 3825.557 & 3828.810 \\
\hline
\end{tabular}
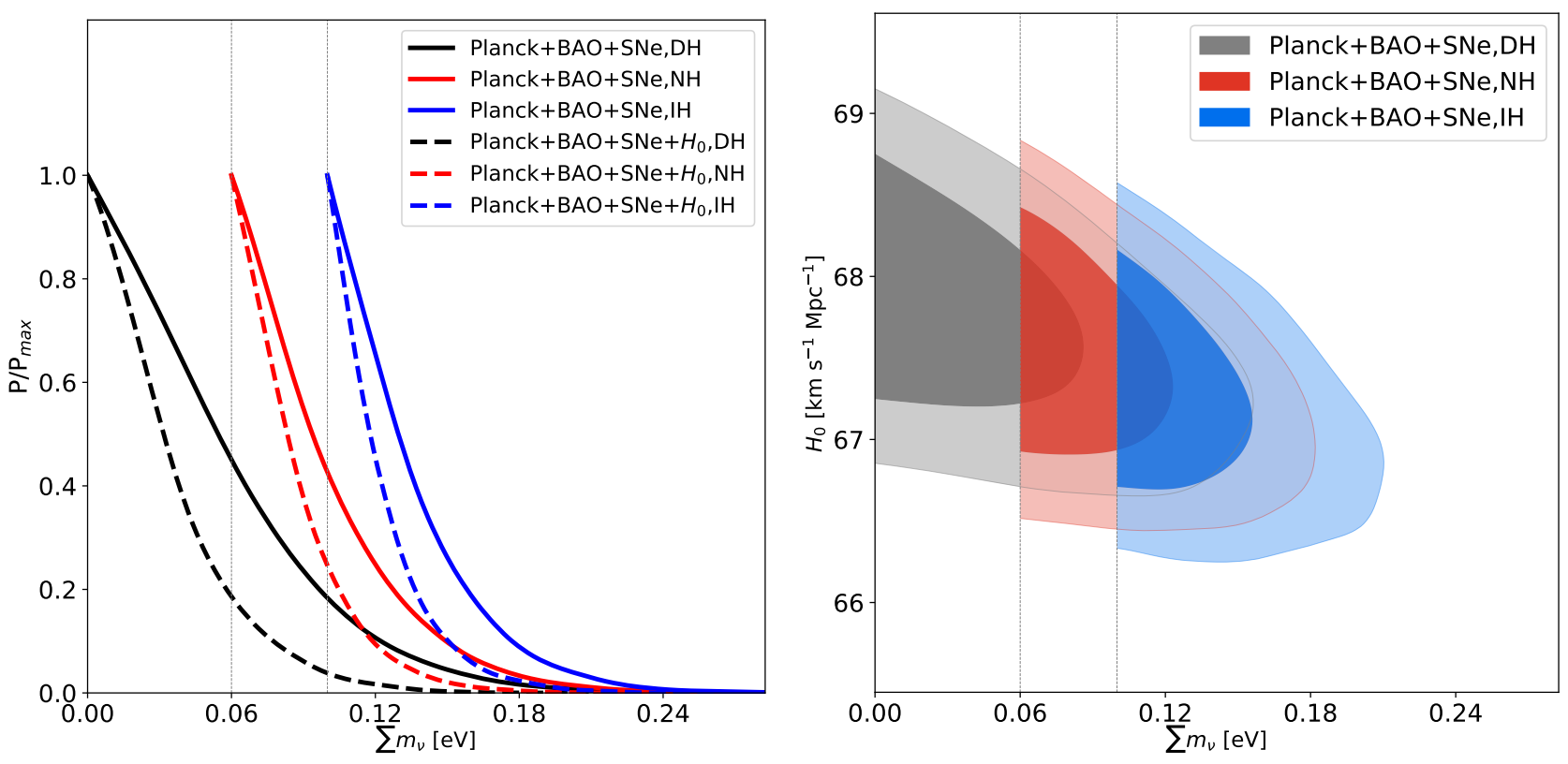

FIG. 1: Left: The one-dimensional marginalized posterior distributions for $\sum m_{\nu}$ using the Planck+BAO+SNe and Planck $+\mathrm{BAO}+\mathrm{SNe}+H_{0}$ data combinations in the $\Lambda \mathrm{CDM}+\sum m_{\nu}$ model. Right: The two-dimensional marginalized contours $(1 \sigma$ and $2 \sigma)$ in the $\sum m_{\nu}-H_{0}$ plane for three neutrino mass hierarchy cases, i.e., the DH case, the NH case, and the IH case, by using Planck+BAO+SNe data combination in the $\Lambda \mathrm{CDM}+\sum m_{\nu}$ model.

which plays an important role in determining the properties of the holographic dark energy. The value of $c$ determines the evolution of $w$. In the HDE model, the EoS can be expressed as

$$
w=-\frac{1}{3}-\frac{2}{3} \frac{\sqrt{\Omega_{\mathrm{de}}}}{c} .
$$

According to this equation, we can find that in the early times $w \rightarrow-1 / 3$ (since $\Omega_{\mathrm{de}} \rightarrow 0$ ) and in the far future $w \rightarrow-1 / 3-2 /(3 c)$ (since $\Omega_{\mathrm{de}} \rightarrow 1$ ). Thus, when $c<1$, we can find that the EoS parameter $w$ crosses -1 during the cosmological evolution. For the $\mathrm{HDE}+\sum m_{\nu}$ model, the parameter space vector is:

$$
P_{4} \equiv\left(\omega_{b}, \omega_{c}, \Theta_{\mathrm{s}}, \tau, n_{\mathrm{s}}, \ln \left[10^{10} \mathrm{~A}_{\mathrm{s}}\right], c, \sum \mathrm{m}_{\nu}\right) .
$$

The observational data sets used in this work include $\mathrm{CMB}, \mathrm{BAO}, \mathrm{SNe}$, and $H_{0}$. Here we also briefly describe these observational data.

- The CMB data: We employ the CMB likelihood including the TT, TE, and EE spectra at $\ell \geq 30$, the low- $\ell$ temperature Commander likelihood, and the low- $\ell$ SimAll EE likelihood, from the Planck 2018 release [11].

- The BAO data: We employ the measurements of the BAO signals from different galaxy surveys, including the DR7 Main Galaxy Sample (MGS) at the effective redshift of $z_{\text {eff }}=0.15$ [65], the Six-degree-Field Galaxy Survey (6dFGS) at $z_{\text {eff }}=$ 0.106 [66], and the latest BOSS data release 12 (DR12) in three redshift slices of $z_{\text {eff }}=0.38,0.51$, and 0.61 [67]. 
TABLE II: Fitting results of the cosmological parameters in the $w \mathrm{CDM}+\sum m_{\nu}$ model for three neutrino mass hierarchy cases, i.e., the DH case, the $\mathrm{NH}$ case, and the $\mathrm{IH}$ case, using the Planck $+\mathrm{BAO}+\mathrm{SNe}$ and Planck $+\mathrm{BAO}+\mathrm{SNe}+H_{0}$ data combinations.

\begin{tabular}{|c|c|c|c|c|c|c|}
\hline Data & \multicolumn{3}{|c|}{ Planck $+\mathrm{BAO}+\mathrm{SNe}$} & \multicolumn{3}{|c|}{ Planck $+\mathrm{BAO}+\mathrm{SNe}+H_{0}$} \\
\hline Mass ordering & $\mathrm{DH}$ & $\mathrm{NH}$ & $\mathrm{IH}$ & $\mathrm{DH}$ & $\mathrm{NH}$ & $\mathrm{IH}$ \\
\hline$w$ & $-1.029 \pm 0.035$ & $-1.042 \pm 0.035$ & $-1.051 \pm 0.035$ & $-1.078 \pm 0.033$ & $-1.090 \pm 0.033$ & $-1.100_{-0.031}^{+0.034}$ \\
\hline$H_{0}\left[\mathrm{~km} \mathrm{~s}^{-1} \mathrm{Mpc}^{-1}\right]$ & $68.27 \pm 0.83$ & $68.23 \pm 0.83$ & $68.21 \pm 0.81$ & $69.79 \pm 0.73$ & $69.74 \pm 0.73$ & $69.70 \pm 0.74$ \\
\hline$\Omega_{\mathrm{m}}$ & $0.3064 \pm 0.0078$ & $0.3076 \pm 0.0078$ & $0.3084 \pm 0.0076$ & $0.2932 \pm 0.0066$ & $0.2945 \pm 0.0066$ & $0.2954 \pm 0.0067$ \\
\hline$\sigma_{8}$ & $0.819 \pm 0.015$ & $0.811_{-0.014}^{+0.015}$ & $0.805 \pm 0.014$ & $0.834_{-0.013}^{+0.015}$ & $0.826_{-0.013}^{+0.014}$ & $0.820 \pm 0.014$ \\
\hline$\sum m_{\nu}[\mathrm{eV}]$ & $<0.155$ & $<0.195$ & $<0.220$ & $<0.145$ & $<0.183$ & $<0.210$ \\
\hline$\chi^{2}$ & 3805.053 & 3806.381 & 3807.724 & 3817.072 & 3818.757 & 3819.912 \\
\hline
\end{tabular}
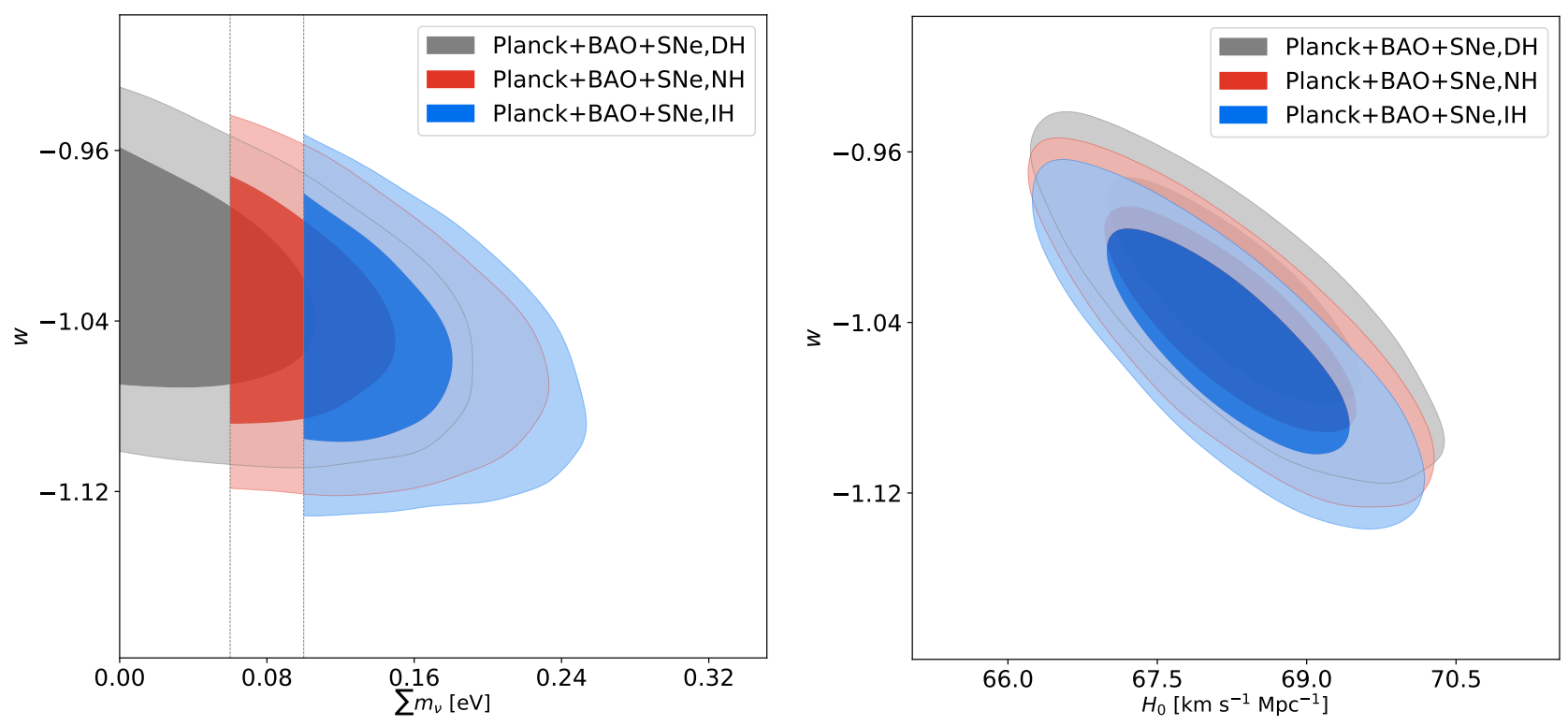

FIG. 2: The two-dimensional marginalized contours $(1 \sigma$ and $2 \sigma)$ in the $\sum m_{\nu}-w$ and $H_{0}-w$ planes for three neutrino mass hierarchy cases, i.e., the $\mathrm{DH}$ case, the $\mathrm{NH}$ case, and the $\mathrm{IH}$ case, by using Planck+BAO+SNe data combination in the $w \mathrm{CDM}+\sum m_{\nu}$ model.

- The SNe data: We use the latest SNe data given the Pantheon Sample [68], which contains 1048 SNe data in the redshift range of $0.01<z<2.3$.

- The Hubble constant: We use the result of the direct measurement of the Hubble constant, with the result of $H_{0}=74.03 \pm 1.42 \mathrm{~km} \mathrm{~s}^{-1} \mathrm{Mpc}^{-1}$, given by Riess et al. [61].

In this study, our basic data combination is Planck+BAO+SNe. In addition, in order to investigate the impacts of the $H_{0}$ measurement on constraints on the neutrino mass, we also consider the data combination of Planck $+\mathrm{BAO}+\mathrm{SNe}+H_{0}$.

\section{RESULTS AND DISCUSSION}

In this section, we report the results of constraining the total neutrino mass from the Planck $+\mathrm{BAO}+\mathrm{SNe}$ and Planck $+\mathrm{BAO}+\mathrm{SNe}+H_{0}$ data combinations. In our analysis, several typical dark energy models, i.e. the $\Lambda \mathrm{CDM}+\sum m_{\nu}$ model, the $w \mathrm{CDM}+\sum m_{\nu}$ model, the $\mathrm{CPL}+\sum m_{\nu}$ model, and the HDE $+\sum m_{\nu}$ model, are investigated. In the meantime, we compare the results of the three neutrino mass hierarchy cases, i.e., the $\mathrm{DH}$ case, the NH case, and the IH case. The main results are listed in Tables I-IV. The best-fit values of $\chi^{2}$ in the various cases are also listed. The fit values of parameters are given at $68 \%$ C.L. $(1 \sigma)$, and the upper limits of the neutrino mass are given at $95 \%$ C.L. $(2 \sigma)$.

\section{A. In different dark energy models}

Firstly, we compare the constraint results in the different dark energy models from the Planck $+\mathrm{BAO}+\mathrm{SNe}$ data combination. In Table I, we can obtain $\sum m_{\nu}<$ $0.123 \mathrm{eV}$ for the $\mathrm{DH}$ case, $\sum m_{\nu}<0.156 \mathrm{eV}$ for the 
TABLE III: Fitting results of the cosmological parameters in the CPL $+\sum m_{\nu}$ model for three neutrino mass hierarchy cases, i.e., the $\mathrm{DH}$ case, the $\mathrm{NH}$ case, and the $\mathrm{IH}$ case, using the Planck $+\mathrm{BAO}+\mathrm{SNe}$ and Planck $+\mathrm{BAO}+\mathrm{SNe}+H_{0}$ data combinations.

\begin{tabular}{|c|c|c|c|c|c|c|}
\hline \multirow{2}{*}{$\frac{\text { Data }}{\text { Mass ordering }}$} & \multicolumn{3}{|c|}{ Planck+BAO+SNe } & \multicolumn{3}{|c|}{ Planck $+\mathrm{BAO}+\mathrm{SNe}+H_{0}$} \\
\hline & $\mathrm{DH}$ & $\mathrm{NH}$ & $\mathrm{IH}$ & $\mathrm{DH}$ & $\mathrm{NH}$ & $\mathrm{IH}$ \\
\hline$w_{0}$ & $-0.945 \pm 0.087$ & $-0.933 \pm 0.089$ & $-0.923 \pm 0.089$ & $-1.003 \pm 0.082$ & $-0.988 \pm 0.086$ & $-0.978 \pm 0.088$ \\
\hline$w_{a}$ & $-0.41_{-0.30}^{+0.44}$ & $-0.52_{-0.32}^{+0.46}$ & $-0.61_{-0.33}^{+0.46}$ & $-0.38_{-0.31}^{+0.41}$ & $-0.50_{-0.33}^{+0.44}$ & $-0.60_{-0.34}^{+0.45}$ \\
\hline$H_{0}\left[\mathrm{~km} \mathrm{~s}^{-1} \mathrm{Mpc}^{-1}\right]$ & $68.22 \pm 0.83$ & $68.19 \pm 0.83$ & $68.14 \pm 0.84$ & $69.78 \pm 0.73$ & $69.71 \pm 0.74$ & $69.69 \pm 0.73$ \\
\hline$\Omega_{\mathrm{m}}$ & $0.3087 \pm 0.0082$ & $0.3102 \pm 0.0083$ & $0.3113 \pm 0.0083$ & $0.2948 \pm 0.0068$ & $0.2965 \pm 0.0070$ & $0.2976 \pm 0.0069$ \\
\hline$\sigma_{8}$ & $0.819_{-0.015}^{+0.018}$ & $0.813_{-0.015}^{+0.018}$ & $0.808_{-0.015}^{+0.018}$ & $0.835_{-0.015}^{+0.017}$ & $0.828_{-0.014}^{+0.017}$ & $0.823_{-0.014}^{+0.017}$ \\
\hline$\sum m_{\nu}[\mathrm{eV}]$ & $<0.247$ & $<0.290$ & $<0.305$ & $<0.216$ & $<0.255$ & $<0.281$ \\
\hline$\chi^{2}$ & 3804.644 & 3805.938 & 3806.531 & 3816.716 & 3817.806 & 3818.809 \\
\hline
\end{tabular}
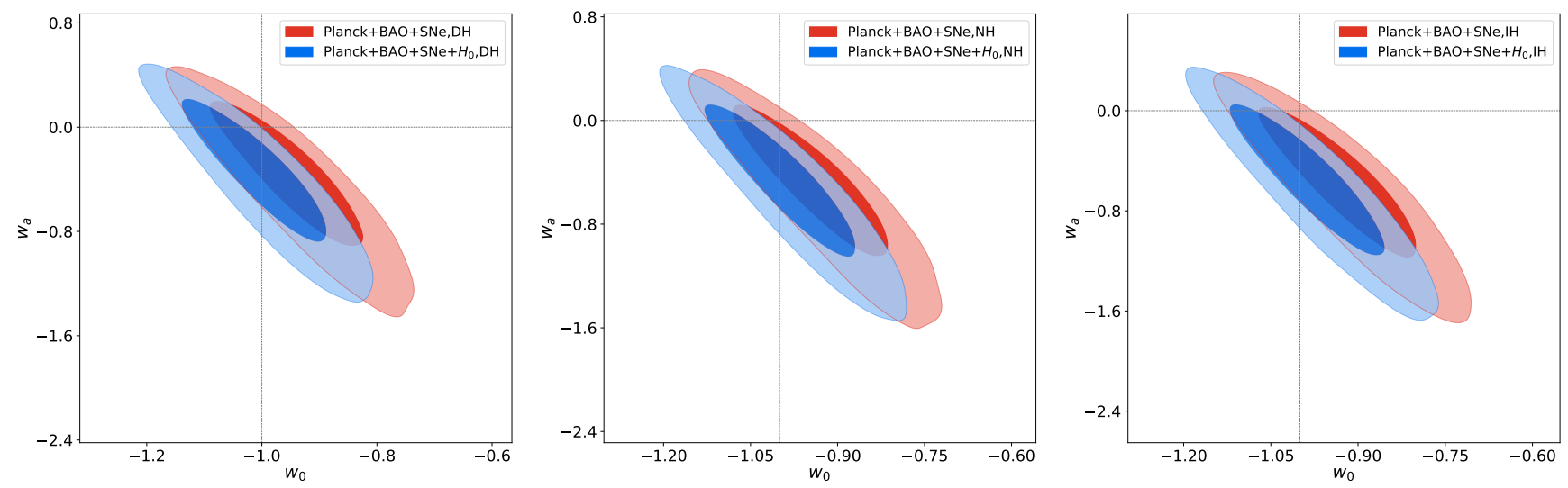

FIG. 3: The two-dimensional marginalized contours $(1 \sigma$ and $2 \sigma)$ in the $w_{0}-w_{a}$ plane for three neutrino mass hierarchy cases, i.e., the $\mathrm{DH}$ case, the $\mathrm{NH}$ case, and the $\mathrm{IH}$ case, by using Planck $+\mathrm{BAO}+\mathrm{SNe}$ and Planck $+\mathrm{BAO}+\mathrm{SNe}+H_{0}$ data combinations in the $\mathrm{CPL}+\sum m_{\nu}$ model.

$\mathrm{NH}$ case, $\sum m_{\nu}<0.185 \mathrm{eV}$ for the $\mathrm{IH}$ case in the $\Lambda \mathrm{CDM}+\sum m_{\nu}$ model. For the $w \mathrm{CDM}+\sum m_{\nu}$ model, we have $\sum m_{\nu}<0.155 \mathrm{eV}(\mathrm{DH}), \sum m_{\nu}<0.195 \mathrm{eV}$ $(\mathrm{NH})$, and $\sum m_{\nu}<0.220 \mathrm{eV}(\mathrm{IH})$ corresponding to $w=-1.029 \pm 0.035(\mathrm{DH}), w=-1.042 \pm 0.035(\mathrm{NH})$, and $w=-1.051 \pm 0.035(\mathrm{IH})$, respectively (see Table II), and we find that the upper limits of $\sum m_{\nu}$ become larger, compared to the $\Lambda \mathrm{CDM}+\sum m_{\nu}$ model. In the $\mathrm{CPL}+\sum m_{\nu}$ model, the neutrino mass bounds are greatly relaxed (see Table III), and they are $\sum m_{\nu}<0.247$ $\mathrm{eV}(\mathrm{DH}), \sum m_{\nu}<0.290 \mathrm{eV}(\mathrm{NH})$, and $\sum m_{\nu}<0.305$ $\mathrm{eV}(\mathrm{IH})$. As is showed in Fig 3, we find that a phantom dark energy (i.e., $w<-1$ ) or an early phantom dark energy (i.e., the quintom evolving from $w<-1$ to $w>-1)$ is slightly more favored by current cosmological observations, which leads to the fact that a larger upper limit of $\sum m_{\nu}$ is obtained in the $w \mathrm{CDM}+\sum m_{\nu}$ and CPL $+\sum m_{\nu}$ models. For the HDE $+\sum m_{\nu}$ model, an early quintessence dark energy with $c<1$ (i.e., the quintom evolving from $w<-1$ to $w>-1$ ) is favored, and we could obtain the most stringent upper limits of the neutrino mass with $\sum m_{\nu}<0.080 \mathrm{eV}(\mathrm{DH}), \sum m_{\nu}<0.129$ $\mathrm{eV}(\mathrm{NH}), \sum m_{\nu}<0.163 \mathrm{eV}(\mathrm{IH})$, as also shown in Ta- ble IV.

In addition, we can compare the best-fit $\chi^{2}$ values of these models, which are listed in Tables I-IV. For the $w \mathrm{CDM}+\sum m_{\nu}$ model, the $\chi^{2}$ values in the same neutrino mass hierarchy are slightly smaller than those of the $\Lambda \mathrm{CDM}+\sum m_{\nu}$ model, at the price of adding one more parameter. We obtain the smallest $\chi^{2}$ values in the $\mathrm{CPL}+\sum m_{\nu}$ model, since this model has the most free parameters. For the HDE $+\sum m_{\nu}$ model, the most stringent upper limits of $\sum m_{\nu}$ can be obtained, but the $\chi^{2}$ values are much larger than those of the $\Lambda \mathrm{CDM}+\sum m_{\nu}$ model.

For all these models, we discuss the fitting results in the different neutrino mass hierarchies. The prior of the lower bounds of $\sum m_{\nu}$ are $0 \mathrm{eV}, 0.06 \mathrm{eV}$ and $0.1 \mathrm{eV}$ for $\mathrm{DH}, \mathrm{NH}$ and $\mathrm{IH}$, respectively, which can affect the constraint results of $\sum m_{\nu}$ significantly. In Table I-IV, the upper limits of $\sum m_{\nu}$ for the $\mathrm{NH}$ case are smaller than those for the IH case in these dark energy models. What's more, we find that the $\chi^{2}$ values in the $\mathrm{NH}$ case is slightly smaller than those in the IH case for all these models, which indicates that the $\mathrm{NH}$ case fits the current observations better than the IH case. This conclusion is 
TABLE IV: Fitting results of the cosmological parameters in the HDE $+\sum m_{\nu}$ model for three neutrino mass hierarchy cases, i.e., the $\mathrm{DH}$ case, the $\mathrm{NH}$ case, and the $\mathrm{IH}$ case, using the Planck $+\mathrm{BAO}+\mathrm{SNe}$ and Planck $+\mathrm{BAO}+\mathrm{SNe}+H_{0}$ data combinations.

\begin{tabular}{|c|c|c|c|c|c|c|}
\hline Data & \multicolumn{3}{|c|}{ Planck $+\mathrm{BAO}+\mathrm{SNe}$} & \multicolumn{3}{|c|}{ Planck $+\mathrm{BAO}+\mathrm{SNe}+H_{0}$} \\
\hline Mass ordering & $\mathrm{DH}$ & $\mathrm{NH}$ & $\mathrm{IH}$ & $\mathrm{DH}$ & $\mathrm{NH}$ & $\mathrm{IH}$ \\
\hline$c$ & $0.645_{-0.031}^{+0.027}$ & $0.632_{-0.030}^{+0.026}$ & $0.623_{-0.029}^{+0.025}$ & $0.608_{-0.025}^{+0.023}$ & $0.595 \pm 0.024$ & $0.587_{-0.024}^{+0.022}$ \\
\hline$H_{0}\left[\mathrm{~km} \mathrm{~s}^{-1} \mathrm{Mpc}^{-1}\right]$ & $67.85 \pm 0.81$ & $67.79 \pm 0.79$ & $67.74 \pm 0.80$ & $69.38 \pm 0.72$ & $69.33 \pm 0.71$ & $69.27 \pm 0.71$ \\
\hline$\Omega_{\mathrm{m}}$ & $0.3061 \pm 0.0077$ & $0.3077 \pm 0.0076$ & $0.3087 \pm 0.0076$ & $0.2927 \pm 0.0065$ & $0.2939 \pm 0.0065$ & $0.2951 \pm 0.0065$ \\
\hline$\sigma_{8}$ & $0.797 \pm 0.013$ & $0.789 \pm 0.013$ & $0.783 \pm 0.013$ & $0.811 \pm 0.013$ & $0.803 \pm 0.012$ & $0.796 \pm 0.12$ \\
\hline$\sum m_{\nu}[\mathrm{eV}]$ & $<0.080$ & $<0.129$ & $<0.163$ & $<0.075$ & $<0.123$ & $<0.159$ \\
\hline$\chi^{2}$ & 3822.977 & 3828.219 & 3830.980 & 3838.467 & 3845.127 & 3845.289 \\
\hline
\end{tabular}
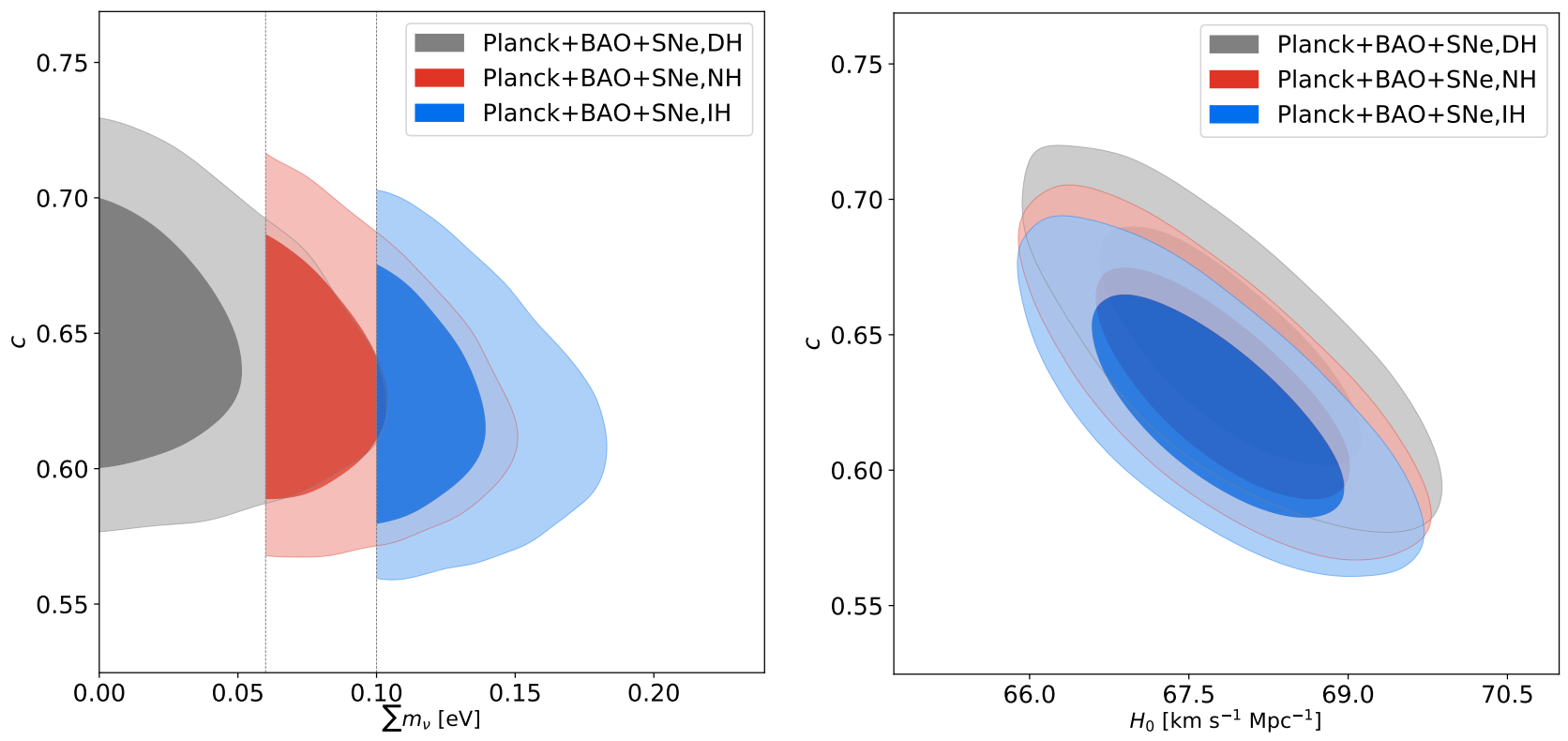

FIG. 4: The two-dimensional marginalized contours $(1 \sigma$ and $2 \sigma)$ in the $H_{0}-c$ and $\sum m_{\nu}-c$ planes for three neutrino mass hierarchy cases, i.e., the DH case, the $\mathrm{NH}$ case, and the IH case, by using Planck+BAO+SNe data combination in the HDE $+\sum m_{\nu}$ model.

still consistent with the previous studies [22, 43, 45, 48, $52,53,59,60]$.

\section{B. Adding the $H_{0}$ measurement in data combination}

In this subsection, we report the constraint results from the $\mathrm{Planck}+\mathrm{BAO}+\mathrm{SNe}+H_{0}$ data combination and investigate the impact of the $H_{0}$ measurement on the fit results of $\sum m_{\nu}$. As is shown in Table I, we have $\sum m_{\nu}<0.082$ $\mathrm{eV}$ for the $\mathrm{DH}$ case, $\sum m_{\nu}<0.125 \mathrm{eV}$ for the $\mathrm{NH}$ case, $\sum m_{\nu}<0.160 \mathrm{eV}$ for the $\mathrm{IH}$ case in the $\Lambda \mathrm{CDM}+\sum m_{\nu}$ model. Adding the $H_{0}$ data leads to a higher $H_{0}$ value in the cosmological fit. From the right panel of Fig 1, we can see that $\sum m_{\nu}$ is anti-correlated with $H_{0}$ in the $\Lambda \mathrm{CDM}+\sum m_{\nu}$ model. Therefore, we obtain a smaller upper limit of $\sum m_{\nu}$ with the Planck $+\mathrm{BAO}+\mathrm{SNe}+H_{0}$ data combination than that with the Planck+BAO+SNe data combination, which can be clearly seen in the left panel of Fig 1.

With the $\mathrm{Planck}+\mathrm{BAO}+\mathrm{SNe}+H_{0}$ data combination, we have $\sum m_{\nu}<0.145 \mathrm{eV}(\mathrm{DH}), \sum m_{\nu}<0.183 \mathrm{eV}$ $(\mathrm{NH}), \sum m_{\nu}<0.210 \mathrm{eV}(\mathrm{IH})$ in the $w \mathrm{CDM}+\sum m_{\nu}$ model (see Table II); we have $\sum m_{\nu}<0.216 \mathrm{eV}(\mathrm{DH})$, $\sum m_{\nu}<0.255 \mathrm{eV}(\mathrm{NH}), \sum m_{\nu}<0.281 \mathrm{eV}$ (IH) in the $\mathrm{CPL}+\sum m_{\nu}$ model (see Table III); we have $\sum m_{\nu}<$ $0.075 \mathrm{eV}(\mathrm{DH}), \sum m_{\nu}<0.123 \mathrm{eV}(\mathrm{NH}), \sum m_{\nu}<0.159$ $\mathrm{eV}(\mathrm{IH})$ in the $\mathrm{HDE}+\sum m_{\nu}$ model (see Table IV). In all these models, we find that the inclusion of the $H_{0}$ data gives a tighter constraint on $\sum m_{\nu}$.

\section{CONCLUSION}

In this paper, using the latest cosmological observations (including the Planck $2018 \mathrm{CMB}$ data), we have obtained $\sum m_{\nu}<0.123 \mathrm{eV}(\mathrm{DH}), \sum m_{\nu}<0.156 \mathrm{eV}$ 
$(\mathrm{NH})$, and $\sum m_{\nu}<0.185 \mathrm{eV}(\mathrm{IH})$ in the $\Lambda \mathrm{CDM}+\sum m_{\nu}$ model with the Planck $+\mathrm{BAO}+\mathrm{SNe}$ data combination. In addition, we also consider the influence of dynamical dark energy on the constraint results of $\sum m_{\nu}$. We investigate the cases of the $w \mathrm{CDM}+\sum m_{\nu}$ model, the CPL $+\sum m_{\nu}$ model, and the $\mathrm{HDE}+\sum m_{\nu}$ model, and we find that the nature of dark energy could significantly affect the constraints on the total neutrino mass. Compared to the $\Lambda \mathrm{CDM}+\sum m_{\nu}$ model, the upper limits of the total neutrino mass become larger in the $w \mathrm{CDM}+\sum m_{\nu}$ and $\mathrm{CPL}+\sum m_{\nu}$ models. Using the Planck $+\mathrm{BAO}+\mathrm{SNe}$ data combination, the most stringent upper limits of the neutrino mass, i.e., $\sum m_{\nu}<0.080 \mathrm{eV}(\mathrm{DH}), \sum m_{\nu}<0.129$ $\mathrm{eV}(\mathrm{NH})$, and $\sum m_{\nu}<0.163 \mathrm{eV}(\mathrm{IH})$, are obtained in the $\mathrm{HDE}+\sum m_{\nu}$ model.

Comparing the values of $\chi^{2}$ between the $\mathrm{NH}$ and $\mathrm{IH}$ cases, it is found that the NH case fits the current cosmological observations better than the IH case, indicating that the neutrino mass hierarchy is more likely to be the
$\mathrm{NH}$ case according to the current cosmological data. In addition, it is also found that the inclusion of the local measurement of the Hubble constant in the data combination will lead to a tighter constraint on the total neutrino mass for all the dark energy model considered in this work.

\section{Acknowledgments}

We thank Hai-Li Li, Jing-Zhao Qi, and Yun-He Li for helpful discussions. This work was supported by the National Natural Science Foundation of China (Grant Nos. 11975072, 11875102, 11835009, and 11690021), the Liaoning Revitalization Talents Program (Grant No. XLYC1905011), the Fundamental Research Funds for the Central Universities (Grant No. N2005030), and the TopNotch Young Talents Program of China.
[1] Y. Fukuda et al. [Super-Kamiokande Collaboration], Phys. Rev. Lett. $81 \quad$ (1998) 1562 doi:10.1103/PhysRevLett.81.1562 [hep-ex/9807003].

[2] Q. R. Ahmad et al. [SNO Collaboration], Phys. Rev. Lett. 89 (2002) 011301 doi:10.1103/PhysRevLett.89.011301 [nucl-ex/0204008].

[3] K. A. Olive et al. [Particle Data Group], Chin. Phys. C 38 (2014) 090001. doi:10.1088/1674-1137/38/9/090001

[4] K. N. Abazajian et al. [Topical Conveners: K.N. Abazajian, J.E. Carlstrom, A.T. Lee Collaboration], Astropart. Phys. $63 \quad$ (2015) 66 doi:10.1016/j.astropartphys.2014.05.014 [arXiv:1309.5383 [astro-ph.CO]].

[5] J. Lesgourgues and S. Pastor, Phys. Rept. 429 (2006) 307 doi:10.1016/j.physrep.2006.04.001 [astro-ph/0603494].

[6] Y. Y. Y. Wong, Ann. Rev. Nucl. Part. Sci. 61 (2011) 69 doi:10.1146/annurev-nucl-102010-130252 [arXiv:1111.1436 [astro-ph.CO]].

[7] J. Lesgourgues and S. Pastor, Adv. High Energy Phys. 2012 (2012) 608515 doi:10.1155/2012/608515 [arXiv:1212.6154 [hep-ph]].

[8] J. Lesgourgues and S. Pastor, New J. Phys. 16 (2014) 065002 doi:10.1088/1367-2630/16/6/065002 [arXiv:1404.1740 [hep-ph]].

[9] M. Archidiacono, T. Brinckmann, J. Lesgourgues and V. Poulin, JCAP 1702 (2017) 052 doi:10.1088/14757516/2017/02/052 [arXiv:1610.09852 [astro-ph.CO]].

[10] M. Lattanzi and M. Gerbino, Front. in Phys. 5 (2018) 70 doi:10.3389/fphy.2017.00070 [arXiv:1712.07109 [astroph.CO]].

[11] N. Aghanim et al. [Planck], [arXiv:1807.06209 [astroph.CO]].

[12] M. Chevallier and D. Polarski, Int. J. Mod. Phys. D 10 (2001) 213 doi:10.1142/S0218271801000822 [grqc/0009008].

[13] E. V. Linder, Phys. Rev. Lett. 90 (2003) 091301 doi:10.1103/PhysRevLett.90.091301 [astro-ph/0208512].

[14] M. Li, Phys. Lett. B $603 \quad$ (2004) 1 doi:10.1016/j.physletb.2004.10.014 [hep-th/0403127].
[15] Q. G. Huang and M. Li, JCAP 0408 (2004) 013 doi:10.1088/1475-7516/2004/08/013 [astro-ph/0404229].

[16] J. F. Zhang, M. M. Zhao, J. L. Cui and X. Zhang, Eur. Phys. J. C 74 (2014) no.11, 3178 doi:10.1140/epjc/s10052-014-3178-7 [arXiv:1409.6078 [astro-ph.CO]].

[17] S. Wang, Y. Wang and M. Li, Phys. Rept. 696 (2017) 1 doi:10.1016/j.physrep.2017.06.003 [arXiv:1612.00345 [astro-ph.CO]].

[18] S. Wang, J. J. Geng, Y. L. Hu and X. Zhang, Sci. China Phys. Mech. Astron. 58 (2015) no.1, 019801 doi:10.1007/s11433-014-5628-5 [arXiv:1312.0184 [astroph.CO]].

[19] J. Cui, Y. Xu, J. Zhang and X. Zhang, Sci. China Phys. Mech. Astron. 58 (2015) 110402 doi:10.1007/s11433-0155734-z [arXiv:1511.06956 [astro-ph.CO]].

[20] D. Z. He, J. F. Zhang and X. Zhang, Sci. China Phys. Mech. Astron. 60 (2017) no.3, 039511 doi:10.1007/s11433-016-0472-1 [arXiv:1607.05643 [astroph.CO]].

[21] Y. Y. Xu and X. Zhang, Eur. Phys. J. C 76 (2016) no.11, 588 doi:10.1140/epjc/s10052-016-4446-5 [arXiv:1607.06262 [astro-ph.CO]].

[22] S. Roy Choudhury and S. Hannestad, [arXiv:1907.12598 [astro-ph.CO]].

[23] B. A. Reid, L. Verde, R. Jimenez and O. Mena, JCAP 1001 (2010) 003 doi:10.1088/1475-7516/2010/01/003 [arXiv:0910.0008 [astro-ph.CO]].

[24] S. A. Thomas, F. B. Abdalla and O. Lahav, Phys. Rev. Lett. $105 \quad$ (2010) 031301 doi:10.1103/PhysRevLett.105.031301 [arXiv:0911.5291 [astro-ph.CO]].

[25] C. Carbone, L. Verde, Y. Wang and A. Cimatti, JCAP 1103 (2011) 030 doi:10.1088/1475-7516/2011/03/030 [arXiv:1012.2868 [astro-ph.CO]].

[26] H. Li and X. Zhang, Phys. Lett. B 713 (2012) 160 doi:10.1016/j.physletb.2012.06.030 [arXiv:1202.4071 [astro-ph.CO]].

[27] Y. H. Li, S. Wang, X. D. Li and X. Zhang, JCAP 
1302 (2013) 033 doi:10.1088/1475-7516/2013/02/033 [arXiv:1207.6679 [astro-ph.CO]].

[28] B. Audren, J. Lesgourgues, S. Bird, M. G. Haehnelt and M. Viel, JCAP 1301 (2013) 026 doi:10.1088/14757516/2013/01/026 [arXiv:1210.2194 [astro-ph.CO]].

[29] S. Riemer-S $\phi$ rensen, D. Parkinson and T. M. Davis, Phys. Rev. D 89 (2014) 103505 doi:10.1103/PhysRevD.89.103505 [arXiv:1306.4153 [astro-ph.CO]].

[30] J. F. Zhang, Y. H. Li and X. Zhang, Phys. Lett. B 740 (2015) 359 doi:10.1016/j.physletb.2014.12.012 [arXiv:1403.7028 [astro-ph.CO]].

[31] J. F. Zhang, Y. H. Li and X. Zhang, Eur. Phys. J. C 74 (2014) 2954 doi:10.1140/epjc/s10052-014-2954-8 [arXiv:1404.3598 [astro-ph.CO]].

[32] J. F. Zhang, J. J. Geng and X. Zhang, JCAP 1410 (2014) 044 doi:10.1088/1475-7516/2014/10/044 [arXiv:1408.0481 [astro-ph.CO]].

[33] N. Palanque-Delabrouille et al., JCAP 1502 (2015) 045 doi:10.1088/1475-7516/2015/02/045 [arXiv:1410.7244 [astro-ph.CO]].

[34] Y. H. Li, J. F. Zhang and X. Zhang, Phys. Lett. B 744 (2015) 213 doi:10.1016/j.physletb.2015.03.063 [arXiv:1502.01136 [astro-ph.CO]].

[35] J. F. Zhang, M. M. Zhao, Y. H. Li and X. Zhang, JCAP 1504 (2015) 038 doi:10.1088/1475-7516/2015/04/038 [arXiv:1502.04028 [astro-ph.CO]].

[36] C. Q. Geng, C. C. Lee, R. Myrzakulov, M. Sami and E. N. Saridakis, JCAP $1601 \quad$ (2016) 049 doi:10.1088/1475-7516/2016/01/049 [arXiv:1504.08141 [astro-ph.CO]].

[37] Y. Chen and L. Xu, Phys. Lett. B 752 (2016) 66 doi:10.1016/j.physletb.2015.11.022 [arXiv:1507.02008 [astro-ph.CO]].

[38] R. Allison, P. Caucal, E. Calabrese, J. Dunkley and T. Louis, Phys. Rev. D 92 (2015) no.12, 123535 doi:10.1103/PhysRevD.92.123535 [arXiv:1509.07471 [astro-ph.CO]].

[39] A. J. Cuesta, V. Niro and L. Verde, Phys. Dark Univ. 13 (2016) 77 doi:10.1016/j.dark.2016.04.005 [arXiv:1511.05983 [astro-ph.CO]].

[40] Y. Chen, B. Ratra, M. Biesiada, S. Li and Z. H. Zhu, Astrophys. J. 829 (2016) no.2, 61 doi:10.3847/0004637X/829/2/61 [arXiv:1603.07115 [astro-ph.CO]].

[41] J. Lu, M. Liu, Y. Wu, Y. Wang and W. Yang, Eur. Phys. J. C 76 (2016) no.12, 679 doi:10.1140/epjc/s10052-0164525-7 [arXiv:1606.02987 [astro-ph.CO]].

[42] S. Kumar and R. C. Nunes, Phys. Rev. D 94 (2016) no.12, 123511 doi:10.1103/PhysRevD.94.123511 [arXiv:1608.02454 [astro-ph.CO]].

[43] L. Xu and Q. G. Huang, Sci. China Phys. Mech. Astron. 61 (2018) no.3, 039521 doi:10.1007/s11433-017-9125-0 [arXiv:1611.05178 [astro-ph.CO]].

[44] S. Vagnozzi, E. Giusarma, O. Mena, K. Freese, M. Gerbino, S. Ho and M. Lattanzi, Phys. Rev. D 96 (2017) no.12, 123503 doi:10.1103/PhysRevD.96.123503 [arXiv:1701.08172 [astro-ph.CO]].

[45] X. Zhang, Sci. China Phys. Mech. Astron. 60 (2017) no.6, 060431 doi:10.1007/s11433-017-9025-7 [arXiv:1703.00651 [astro-ph.CO]].

[46] X. Zhang, Phys. Rev. D 93 (2016) no.8, 083011 doi:10.1103/PhysRevD.93.083011 [arXiv:1511.02651 [astro-ph.CO]].

[47] C. S. Lorenz, E. Calabrese and D. Alonso, Phys. Rev. D
96 (2017) no.4, 043510 doi:10.1103/PhysRevD.96.043510 [arXiv:1706.00730 [astro-ph.CO]].

[48] M. M. Zhao, J. F. Zhang and X. Zhang, Phys. Lett. B 779 (2018) 473 doi:10.1016/j.physletb.2018.02.042 [arXiv:1710.02391 [astro-ph.CO]].

[49] S. Vagnozzi, S. Dhawan, M. Gerbino, K. Freese, A. Goobar and O. Mena, Phys. Rev. D 98 (2018) no.8, 083501 doi:10.1103/PhysRevD.98.083501 [arXiv:1801.08553 [astro-ph.CO]].

[50] L. F. Wang, X. N. Zhang, J. F. Zhang and X. Zhang, Phys. Lett. B 782 (2018) 87 doi:10.1016/j.physletb.2018.05.027 [arXiv:1802.04720 [astro-ph.CO]].

[51] J. F. Zhang, B. Wang and X. Zhang, Sci. China Phys. Mech. Astron. 63 (2020) no.8, 280411 doi:10.1007/s11433-019-1516-y [arXiv:1907.00179 [astroph.CO]].

[52] R. Y. Guo, J. F. Zhang and X. Zhang, Chin. Phys. C 42 (2018) no.9, 095103 doi:10.1088/1674-1137/42/9/095103 [arXiv:1803.06910 [astro-ph.CO]].

[53] M. Zhao, R. Guo, D. He, J. Zhang and X. Zhang, Sci. China Phys. Mech. Astron. 63 (2020) no.3, 230412 doi:10.1007/s11433-019-1474-8 [arXiv:1810.11658 [astroph.CO]].

[54] A. Loureiro et al., Phys. Rev. Lett. 123 (2019) no.8, 081301 doi:10.1103/PhysRevLett.123.081301 [arXiv:1811.02578 [astro-ph.CO]].

[55] Q. G. Huang, K. Wang and S. Wang, Eur. Phys. J. C 76 (2016) no.9, 489 doi:10.1140/epjc/s10052-016-4334-z [arXiv:1512.05899 [astro-ph.CO]].

[56] N. Bellomo, E. Bellini, B. Hu, R. Jimenez, C. Pena-Garay and L. Verde, JCAP 02 (2017), 043 doi:10.1088/14757516/2017/02/043 [arXiv:1612.02598 [astro-ph.CO]].

[57] B. Hu and J. Torrado, Phys. Rev. D 91 (2015) no.6, 064039 doi:10.1103/PhysRevD.91.064039 [arXiv:1410.4804 [astro-ph.CO]].

[58] L. Xu, JCAP 08 (2016), 059 doi:10.1088/14757516/2016/08/059 [arXiv:1605.02403 [astro-ph.CO]].

[59] S. Wang, Y. F. Wang, D. M. Xia and X. Zhang, Phys. Rev. D 94 (2016) no.8, 083519 doi:10.1103/PhysRevD.94.083519 [arXiv:1608.00672 [astro-ph.CO]].

[60] L. Feng, H. L. Li, J. F. Zhang and X. Zhang, Sci. China Phys. Mech. Astron. 63 (2020) no.2, 220401 doi:10.1007/s11433-019-9431-9 [arXiv:1903.08848 [astroph.CO]].

[61] A. G. Riess, S. Casertano, W. Yuan, L. M. Macri and D. Scolnic, Astrophys. J. 876 (2019) no.1, 85 doi:10.3847/1538-4357/ab1422 [arXiv:1903.07603 [astroph.CO]].

[62] A. Lewis and S. Bridle, Phys. Rev. D 66 (2002), 103511 doi:10.1103/PhysRevD.66.103511 [arXiv:astroph/0205436 [astro-ph]].

[63] A. Lewis, A. Challinor and A. Lasenby, Astrophys. J. 538 (2000) 473 doi:10.1086/309179 [astro-ph/9911177].

[64] A. G. Cohen, D. B. Kaplan and A. E. Nelson, Phys. Rev. Lett. 82 (1999) 4971 doi:10.1103/PhysRevLett.82.4971 [hep-th/9803132].

[65] A. J. Ross, L. Samushia, C. Howlett, W. J. Percival, A. Burden and M. Manera, Mon. Not. Roy. Astron. Soc. 449 (2015) no.1, 835 doi:10.1093/mnras/stv154 [arXiv:1409.3242 [astro-ph.CO]].

[66] F. Beutler et al., Mon. Not. Roy. Astron. Soc. 416 (2011) 3017 doi:10.1111/j.1365-2966.2011.19250.x 
[arXiv:1106.3366 [astro-ph.CO]].

[67] S. Alam et al. [BOSS Collaboration], Mon. Not. Roy. Astron. Soc. 470 (2017) no.3, 2617 doi:10.1093/mnras/stx721 [arXiv:1607.03155 [astroph.CO]].
[68] D. M. Scolnic et al., Astrophys. J. 859 (2018) no.2, 101 doi:10.3847/1538-4357/aab9bb [arXiv:1710.00845 [astroph.CO]]. 